


\section{REVISTA DE PSICOLOGÍA}

Vol. XXIII. Segundo semestre 2005 Nº 2

\section{CONTENIDO}

\section{ARTÍCULOS}

Denise Benatuil, Alejandro Castro Solano y Alejandro Torres. Inteligencia práctica: un instrumento para su evaluación.

Dora Herrera Paredes y Hugo Morales Córdova. Comportamiento antisocial durante la adolescencia: teoría, investigación y programas de prevención.

James S. Fleming y César Merino Soto. Medidas de simplicidad y de ajuste factorial: un enfoque para la evaluación de escalas construidas factorialmente.

Cecilia Chau y Stephan Van den Broucke. Consumo de alcohol y sus determinantes en estudiantes universitarios limeños: estudio de focus group.

Inés Kudó, Tesania Velásquez, Mónica Iza, Alicia Ángeles, César Pezo del Pino y Patricia Martínez U. Una experiencia de intervención en crisis en el sur del Perú: Brigadas Psicológicas. 
Revista de Psicología de la PUCP. Vol. XXIII, 2, 2005

\title{
Inteligencia práctica: un instrumento para su evaluación ${ }^{1}$
}

\author{
Denise Benatuil ${ }^{2}$, Alejandro Castro Solano ${ }^{3}$ y Alejandro Torres ${ }^{4}$ \\ Colegio Militar de la Nación, Argentina
}

En este estudio se presentan los pasos para la construcción de un instrumento para evaluar la inteligencia práctica -conocimiento tácito- basado en la teoría de R. Sternberg (Sternberg et al., 2000). Para ello se trabajó en diferentes etapas y con distintos instrumentos. La primera fue la detección de incidentes críticos a partir de entrevistas semidirigidas a 25 cadetes que realizan un programa de entrenamiento combinado académico/militar. La segunda consistió en la aplicación del instrumento piloto (CTLM) a 150 oficiales militares, egresados recientes, construido a partir de los incidentes críticos detectados. La tercera consistió en la evaluación de la calidad de los ítems mediante la técnica de focus group por parte de 10 oficiales instructores -expertos a cargo de la formación de cadetes. En la cuarta fase se diseñaron los tipos de respuestas esperadas para la resolución del incidente, según el criterio de los oficiales instructores y las respuestas específicas de los cadetes a la versión piloto del instrumento CTLM.

Palabras clave: inteligencia práctica, conocimiento tácito, construcción de instrumentos.

\section{Practical intelligence: An instrument for its assessment}

This report presents the steps for the construction of an instrument to asses practical intelligence -tacit knowledge- based on Sternberg's theory (Sternberg et al., 2000). The process had different stages and different instruments were used. The first step was the detection of critical incidents starting from in depth-interviews to 25 cadets who were in a military training. The second consisted on the administration of the first version of the instrument (CTLM) to 150 freshman military officers. This version was designed taking into account the critical incidents identified in step one. In the third step 10 military officers (experts in military training) gathered in a focus group, assessed the quality of the tacit knowledge items. In the last stage, key answers were designed for optimal incident resolution, taking into account officers' criteria and cadets' answers to pilot version of the instrument.

Keywords: practical intelligence, tacit knowledge, instrument construction.

1 Publicación preliminar de la Tesis Doctoral de la primera autora. Proyecto subsidiado por la Secretaría de Ciencia, Tecnología e Innovación Productiva de la Nación (Argentina). SECTIP 0242/03.

2 Licenciada en Psicología, Universidad de Buenos Aires. Profesora de la Facultad de Ciencias Sociales, Universidad de Palermo. Correo electrónico: dbenatuil@ iname.com 

La inteligencia ha sido uno de los constructos más antiguos, polémicos y con mayor desarrollo dentro de la psicología. Originariamente el concepto de facultad mental provino de la filosofía aristotélica, posteriormente dentro de la medicina surge la craneometría y tempranamente despertó el interés de los psicólogos (Andrés Pueyo, 1997). Aún en nuestros días la inteligencia es una de las fuentes de diferencias individuales más investigadas, tanto desde el punto de vista académico como clínico.

En 1921 un panel de expertos especialmente consultados para determinar qué era la inteligencia llegó a la conclusión de que se trataba de la capacidad para adaptarse exitosamente al ambiente. Los autores hacían un fuerte énfasis en los procesos mentales básicos y de orden superior tales como la resolución de problemas y la toma de decisiones (Sternberg, 2000). Las definiciones señalaban que esta capacidad era generalmente abstracta y que constaba de ciertos procesos mentales que daban cuenta de los comportamientos inteligentes.

En 1986 un simposio similar realizado con el propósito de revisar los acuerdos y discrepancias entre los teóricos en los últimos 65 años otorgaba mucho más importancia a los procesos cognitivos básicos. La inteligencia tenía que ver con procesos tales como la metacognición y con las funciones ejecutivas. Asimismo,

3 Doctor en Psicología, Universidad Complutense de Madrid. Investigador Adjunto del CONICET, Argentina. Investigador responsable del proyecto "El desarrollo de las competencias profesionales de estudiantes militares", Ministerio de Defensa, Ejército Argentino. Correo electrónico: alito@ fibertel.com.ar

4 Licenciado en Educación. Secretario de Investigaciones del Colegio Militar de la Nación, Ministerio de Defensa, Ejercito Argentino. 
comenzó a considerarse el papel de la cognición situada y de las diferencias culturales (Sternberg \& Detterman, 1992).

En sus inicios el campo de la inteligencia era en su mayoría ateórico, estaba centrado en la medición de las diferencias individuales y en la exactitud de los instrumentos de evaluación. A finales del siglo XX, en cambio, el acento estaba puesto más en los procesos del funcionamiento mental que en los productos. La inteligencia dejó de considerarse un constructo fijo para la predicción del rendimiento educativo y/o laboral para ser una capacidad maleable que podía entrenarse. Asimismo, los comportamientos inteligentes variaban bastante según el contexto de aplicación y la tarea por resolver (Sternberg, Lautrey \& Lubart, 2003).

Debido a que el campo de la inteligencia es amplio y diverso, se requiere agrupar las teorías en modelos. Si bien hay infinidad de modos para organizar las teorías, se utilizará la clasificación que realizan Davidson y Downing (2000). La misma se compone de 4 modelos (Ver Cuadro 1).

\section{Cuadro 1}

Modelos de la inteligencia (Davidson \& Downing, 2000)

\begin{tabular}{lll}
\hline Modelo & Autores & Postulado \\
\hline $\begin{array}{l}\text { Eficiencia } \\
\text { Neural }\end{array}$ & Vernon y Jensen. & La inteligencia se encuentra en el cerebro. \\
Jerárquicos & $\begin{array}{l}\text { Spearman, Cattell, } \\
\text { Carroll y Thurstone. }\end{array}$ & $\begin{array}{l}\text { Concepto de "g", conjuntos de habilidades } \\
\text { mentales o estratos. }\end{array}$ \\
$\begin{array}{l}\text { Contextuales } \\
\text { Complejos }\end{array}$ & $\begin{array}{l}\text { Gerry y Bennett. } \\
\text { Gardner, Ceci, }\end{array}$ & $\begin{array}{l}\text { La inteligencia varía de una cultura a otra. } \\
\text { La inteligencia combina aspectos de los } \\
\end{array}$ \\
& Salovey y Goleman. & \\
\hline
\end{tabular}

El modelo de la eficiencia neural comprende al conjunto de teorías que consideran que el centro de la inteligencia es el cerebro. 
Para comprenderla y medirla, se deben estudiar las bases neurofisiológicas de las habilidades mentales. Los autores que adhieren a este modelo parten de la premisa de que las personas con elevados niveles de inteligencia tienen grados más altos de eficiencia neuronal. Este modelo utiliza dos métodos de estudio: 1) el análisis de los potenciales evocados y la tasa de metabolización cerebral de la glucosa, y 2) la comparación de distintos niveles de CI y diferentes grados de eficiencia neuronal. Una de las críticas centrales que se ha recibido es la reducción del constructo inteligencia a un fenómeno de naturaleza biológica. La ventaja de este modelo es que resulta "culturalmente justo", restando peso a los factores culturales y educativos. Si la inteligencia es equiparable a neurofisiología, entonces las diferencias culturales y educativas tendrían poca influencia en los comportamientos inteligentes (Davidson \& Downing, 2000).

Los modelos jerárquicos proponen que la estructura de la inteligencia puede ser aprehendida mediante el análisis de las relaciones entre las puntuaciones obtenidas en diversos tests de habilidades mentales. Las primeras teorías psicométricas han sido las de Spearman y Thurstone. Dentro de este paradigma hay otras dos teorías que han sido de gran relevancia, la teoría de Cattell (1943, 1963) que propone una inteligencia general compuesta por la inteligencia fluida (gf) y la cristalizada (gc), y el modelo de los tres estratos de Carroll (1993, 1996). El autor concibe la inteligencia como una pirámide de tres niveles, donde el tercer nivel se corresponde con "g" sobre el cual subyace la actividad intelectual. Si bien los modelos comentados tienen una base empírica fuerte, existen serias dudas sobre qué es lo que "g" representa.

Los modelos contextuales plantean que aquello que es considerado como comportamiento inteligente en una cultura puede no serlo en otra (Berry \& Bennett, 1992; Das, 1994). Alude a las variaciones émicas del constructo inteligencia. Por consiguiente, si 
la inteligencia tiene grandes variaciones en función del contexto, entonces no puede ser evaluada mediante los tests convencionales. Estas teorías consideran que la inteligencia tiene un significado específico dentro de cada contexto cultural, y que el desarrollo intelectual ocurre y toma forma dentro de un contexto cultural y ecológico con leyes particulares (Irvine \& Berry, 1986). Los modelos contextuales están basados en una gran cantidad de estudios empíricos que demuestran la importancia que tienen los elementos ambientales en la performance del individuo. Si bien estos modelos fueron muy útiles para cuestionar la concepción estática y universal del CI, aún no está claramente definido cuándo y cómo integrar los elementos contextuales a las investigaciones sobre la inteligencia.

Los modelos complejos están caracterizados por combinar aspectos de las teorías biológicas, jerárquicas y contextuales. Incluyen la interacción entre los procesos mentales, las influencias contextuales y las habilidades múltiples (Davidson \& Downing, 2000). Dentro de éstos se encuentran la Teoría de las Inteligencias Múltiples de Gardner (1983, 1998), el Modelo Bioecológico de Ceci (1996), la Teoría Triarquica de R. Sternberg (1985, 1988, 1997a) y la Teoría de la Inteligencia Emocional (Mayer, Salovey \& Caruso, 2000).

En términos generales los debates contemporáneos están vinculados a la existencia de una o varias inteligencias, la interrelación entre cognición y personalidad, el cambio en la concepción del CI y su aplicación a diferentes ámbitos, la modificabilidad de la inteligencia y los avances biológicos y su contribución al entendimiento de la inteligencia. 


\section{Evaluación de la inteligencia}

Las primeras teorías de la inteligencia despertaron rápidamente el interés por la medición y evaluación del constructo. Dos hechos desencadenaron este interés particular: la educación universal obligatoria y la creencia de que la psicología podía llegar a ser una ciencia cuantitativa (Thorndike, 1997). Desde aquel momento se han desarrollado una gran cantidad de teorías de la inteligencia, muchas de ellas con sus correspondientes instrumentos para su medición.

El inicio de la evaluación de la inteligencia se puede ubicar a finales del siglo XIX, época en la cual Galton propuso la teoría de la habilidad mental humana. El autor consideraba que las reglas generales de la conducta podrían conocerse estudiando las diferencias individuales y pensaba que los rasgos humanos eran heredados proponiéndose medir la capacidad mental innata. Galton fue el primero en decir que las facultades mentales podían evaluarse cuantitativamente (Myers, 1997). Catell acuñó el término tests mentales y fue el primero en proponer que existían habilidades mentales que podían medirse objetivamente mediante los tests (Ittenbach, Esters \& Wainer, 1997).

Binet sostenía una postura opuesta a la de Galton, ya que desestimaba la idea de medir múltiples habilidades sencillas y aseguraba que la inteligencia debía estudiarse a partir de procesos mentales complejos. En 1904 Binet creó un instrumento que se deriva de esta concepción de la inteligencia (Brody, 2000).

En 1904 se presentó por primera vez en Francia la escala Binet-Simon, que consistía en 30 tareas breves presentadas en orden de dificultad creciente. La misma había sido diseñada a pedido del gobierno francés para poder evaluar a los niños al momento de su ingreso al sistema escolar. Para la misma época Blin 
y Damaye habían creado un conjunto de preguntas para detectar el retraso mental (Wolf, 1973). Pocos años después, en 1911, se publicaron los primeros estudios de Terman, realizados en Estados Unidos con 400 chicos, utilizando la escala de Binet en su versión norteamericana (Stanford-Binet).

Si bien Binet planteó que su prueba evaluaba el rendimiento académico y no era una medida global de la inteligencia, con el paso de los años se le fue dando un uso mucho más generalizado que el que originariamente se había propuesto el autor.

Entre los años 40 y 50 en Estados Unidos se popularizó el uso de los tests y aparecieron la mayor parte de los instrumentos que se utilizan actualmente. El Test de Matrices Progresivas de Raven, la Batería de Habilidades Mentales Primarias de Thurstone, el Test de Aptitudes Diferenciales (DAT), el Test de Clasificación de Aptitudes de Flanagan y la Escala de Inteligencia para Adultos de Wechsler (WAIS), entre otros. La mayoría de los tests mencionados continúan utilizándose en la actualidad con sus correspondientes revisiones y adaptaciones.

Desde su creación hasta la actualidad, las pruebas para evaluar la inteligencia han recibido varias críticas; una de las principales es que favorecen a los niños y adultos blancos, de clase media y por ello se plantea que es absurdo evaluar con pruebas similares a personas con marcadas diferencias culturales y sociales (Gross, 1998). Los tests que evalúan el CI tienen un gran componente cultural y están muy relacionados con el nivel educativo de las personas (Neisser et al., 1996).

Herrnstein y Murray (1996), en su famoso y polémico libro The Bell Curve, manifiestan que es mejor nacer inteligente que rico, ya que la inteligencia (considerando por tal la medición de "g") es un predictor importante del nivel de ocupación alcanzado 
en la edad adulta y del nivel de ingresos. "g" es el mejor predictor para obtener un buen empleo porque los mejores trabajos exigen entrenarse continuamente y aprender conocimiento nuevo de forma constante, que es precisamente aquello que garantiza "g". Otros predictores tales como la experiencia, la personalidad y las habilidades psicomotrices son mucho más débiles. Aquella persona que posea un nivel mayor de "g" puede aprender más rápidamente de la experiencia y tener éxito en cualquier programa de entrenamiento laboral (Gottfredson , 1997).

Las pruebas de inteligencia pueden dividirse en dos categorías: las pruebas de aptitud también conocidas como ability (predicen la capacidad para aprender una nueva habilidad o tarea) y las de logro conocidas como achievement (evalúan cuánto sabe una persona sobre una temática específica). Estas últimas suelen utilizarse con fines educativos y/o laborales. Un ejemplo de estas últimas es el SAT, creado en 1923 y utilizado predominantemente para el ingreso a la universidad (Anastasi, 1998). Muchos creadores de pruebas de CI las clasifican como pruebas de aptitud. También hay quienes sostienen que hoy las pruebas de logro y las de CI se diferencian solo por el grado de instrucción requerido para poder realizarlas.

Con el paso del tiempo, el público y los usuarios entendieron que los tests medían cosas para las cuales no fueron construidos. Sin embargo, a pesar de las fuertes críticas que han sufrido, los tests clásicos continúan utilizándose en todos los ámbitos profesionales: clínico, educativo, laboral y jurídico.

Actualmente algunos autores afirman que tener una buena puntuación en las pruebas clásicas de inteligencia no lleva necesariamente a tener un desarrollo exitoso en la vida (Sternberg, 1997). Estos hallazgos han llevado a ampliar las concepciones sobre la inteligencia, considerándola más compleja y abarcativa que 
el CI. Si bien se han generado muchos modelos modernos para explicar la inteligencia, éstos no han sido acompañados por los correspondientes instrumentos para su evaluación.

\section{Inteligencia práctica}

La teoría de la inteligencia práctica propuesta por R. Sternberg (1997 op.cit.), es considerada una de las teorías modernas de la inteligencia. La misma ha tenido gran difusión, pero aún no se han popularizado los instrumentos para su evaluación.

Dentro de la teoría triárquica (Sternberg, 1997ª) la Inteligencia práctica (IP) corresponde a la subteoría contextual. La misma implica la aplicación práctica en el mundo real de los metacomponentes (procesos complejos tales como seleccionar el problema a resolver, los subcomponentes que se usarán, el modo para organizar la información, la estrategia para combinarlos, el control de la solución y la sensibilidad para la retroalimentación externa), como así también la adquisición de conocimientos y componentes de desempeño específicos.

Wagner (1994) la define como una aptitud para resolver problemas poco estructurados, que surgen en la vida cotidiana y para los cuales no existe una solución clara y explícita.

La IP es definida como la habilidad para adaptarse, modelar y seleccionar diariamente el entorno. Se puede evaluar a través del conocimiento tácito -know how- (Sternberg, Wagner, Williams \& Horvath, 1995; Wagner \& Sternberg, 1985). Es por naturaleza procedural, se adquiere con poca intervención de los otros, está muy vinculada a la acción y aumenta con la edad y la adquisición de experiencia (Forsythe et al., 1998; Wagner, 1987). La IP no es reductible a reglas explícitas, depende del contexto y se codifica 
en la memoria episódica. Si bien aumenta con la experiencia no es directamente proporcional a la cantidad de experiencia adquirida, generalmente no correlaciona con la inteligencia general (CI) (Wagner, 1987 op.cit.; Sternberg, Wagner \& Okagaki, 1993). En algunos dominios ha demostrado ser independiente de otros predictores de éxito (como CI, personalidad ó estilo cognitivo) (Sternberg \& Wagner, 1993).

Sternberg (1997) propone que los resultados en evaluaciones de inteligencia práctica conjuntamente con la creativa son al menos tan buenos predictores del éxito en la vida como los puntajes de los tests clásicos (inteligencia analítica).

La inteligencia práctica no puede ser considerada de forma aislada del contexto en el cual se la quiere medir (Berg \& Calderone, 1994). Primero, porque diferentes culturas tienen diferentes concepciones de la inteligencia. Por otra parte, la teoría triárquica de la inteligencia es una teoría con gran influencia cultural, por ello es verdaderamente relevante que los instrumentos para su evaluación sean adecuados a los diferentes medios socioculturales.

Una de las críticas que realiza Sternberg a los tests clásicos es que la inteligencia no está libre de influencias ambientales y que no se puede medir de forma descontextualizada.

\section{La evaluación del conocimiento tácito}

El conocimiento tácito se evalúa a partir del examen de las competencias relevantes para tener una buena performance en la vida real. Para ello Sternberg revisa tres modalidades posibles de evaluación: la técnica de incidentes críticos de Flanagan, las situaciones de simulación y los tests de juicio situacional (Sternberg et 
al., 2000). La técnica de incidentes críticos (Flanagan, 1954) intenta identificar aquellas conductas que están asociadas con un desempeño (performance) efectivo. El incidente crítico describe una conducta, el escenario en el que la misma ocurre y sus consecuencias. Los incidentes críticos se detectan a partir de las conductas efectivas y poco efectivas en distintas situaciones laborales. Boyatzis (1982) introduce algunas variantes al método de Flanagan al introducir la entrevista de eventos conductuales, donde se generan incidentes críticos de personas identificadas previamente como de buena, media y baja performance. El autor propone examinar los incidentes obtenidos por los grupos de alto y de bajo rendimiento.

Los incidentes críticos pueden surgir de entrevistas realizadas a expertos o de observación de situaciones de trabajo o entrenamiento reales. Luego de un análisis cualitativo se determina la naturaleza de las competencias que aparecen como importantes para tener éxito en una determinada tarea.

Una de las limitaciones que tiene esta metodología consiste en suponer que las personas pueden describir incidentes críticos que den lugar al desempeño exitoso en su trabajo (Sternberg et al., 2000).

El conocimiento tácito está basado en el nivel de dominio específico que los individuos han adquirido para solucionar los problemas cotidianos de su ámbito laboral. Se basa en la existencia de diferencias en cuanto a la cantidad y modalidad de organización del conocimiento entre expertos y novatos y en el supuesto de que estas diferencias se reflejan en el desempeño entre ambos grupos (Sternberg, 1998).

Sternberg sugiere tener en cuenta algunos elementos para la construcción de un instrumento de conocimiento tácito (Sternberg et al., 2000). A continuación se reseñan los principios recomendados: 
1. Revisión de la literatura y manuales específicos.

2. Realización de entrevistas en profundidad.

3. Selección y codificación de los ítems de conocimiento tácito.

4. Clasificación de los ítems.

5. Creación de categorías.

6. Construcción y desarrollo del inventario preliminar.

7. Feedback de los expertos sobre el inventario.

8. Realización de focus group.

9. Administración de la versión piloto del inventario.

10. Análisis estadístico de los datos obtenidos.

11. Selección de los ítems definitivos.

Tres preguntas guiaron la presente investigación: ¿es posible identificar el conocimiento tácito de los líderes militares? De ser identificable ¿se puede medir? En ese caso, ¿poseer ese conocimiento tácito marca una diferencia en la calidad del desempeño de los líderes? En la fase actual del proyecto sólo intentaremos responder a la primera pregunta, reseñando los pasos 1 al 9 comentados. En consecuencia este trabajo tiene como objetivos:

1. Detectar los indicadores de conocimiento tácito vinculado al liderazgo militar en población militar de cadetes.

2. Construir un instrumento para evaluar el conocimiento tácito en población militar de cadetes.

\section{Metodología}

\section{Participantes}

El estudio está conformado por tres muestras. La primera compuesta por 25 estudiantes (cadetes) que cursaban el último año en una institución militar perteneciente a las Fuerzas Armadas Argentinas. 15 cadetes tenían alto rendimiento académico y militar, 
5 rendimiento académico medio y militar alto, y 5 rendimiento académico bajo y militar promedio. Estos jóvenes realizaban un programa de entrenamiento combinado académico/militar de 4 años de duración. La muestra estuvo compuesta predominantemente por varones con un promedio de edad de 22,01 ( $D E=$ 1.90).

La segunda muestra estuvo formada por 150 oficiales militares subalternos (subtenientes), predominantemente varones. Se trataba de egresados recientes de una institución militar perteneciente a las Fuerzas Armadas Argentinas. El promedio de edad fue de 24,03 (DE =1.80).

La tercera estaba compuesta de 10 oficiales militares instructores, expertos a cargo de la formación de los cadetes, en este caso fue población masculina. En las tres muestras la participación fue voluntaria. Estos participantes eran oficiales subalternos que tenían a cargo el entrenamiento militar de los cadetes militares.

\section{Instrumentos}

Para este estudio se utilizaron dos instrumentos: a) Entrevistas individuales, las cuales tuvieron un formato semidirigido y todas fueron grabadas con el consentimiento de los participantes. Todos los cadetes que participaron de ellas habían tenido experiencias previas de conducción grupal. b) Posteriormente, se trabajó con una versión piloto del instrumento Conocimiento Tácito del Liderazgo Militar (CTLM). El mismo fue construido por los autores del artículo mediante el procedimiento que se detalla a continuación. La versión utilizada consta de 35 situaciones simuladas en formato de lápiz y papel, de respuesta abierta. 


\section{Procedimiento}

En el presente trabajo se utilizó la metodología de incidentes críticos descrita anteriormente (Boyatzis, 1982; Flanagan, 1954). Se comenzó administrando las entrevistas semidirigidas. La consigna era que los estudiantes relataran aquellas experiencias que les habían servido para conducir o liderar grupos y que no estaban escritas en los libros, ni las habían aprendido en las materias del ciclo de formación. Las mismas tenían por finalidad obtener información vinculada al conocimiento tácito, para ello se indagó a profundidad el recuerdo de situaciones reales que les hubieran sucedido durante su entrenamiento académico y militar.

De las historias obtenidas, se relevaron las situaciones que les sirvieron a los cadetes para liderar grupos y se sintetizaron los temas principales. Se generaron a partir de las mismas 10 dimensiones centrales para el conocimiento tácito del liderazgo. A partir de la identificación de estas situaciones prototípicas se construyeron las situaciones simuladas(escenarios) que son la base del instrumento propuesto. Se aplicó para esta codificación la técnica de análisis de contenido, mediante la generación de categorías empíricas (Sampieri, Collado \& Fernández, 1998).

Paralelamente, se realizaron observaciones de situaciones de entrenamiento simuladas, las mismas sirvieron para comprender mejor las entrevistas, tener una aproximación a las situaciones relatadas y terminar de redactar los escenarios.

Posteriormente, se procedió a la construcción de un instrumento de Conocimiento Tácito (CTLM). Se redactaron 37 escenarios correspondientes a las 10 dimensiones antes mencionadas. Estos escenarios fueron inicialmente evaluados por un grupo de 10 cadetes de tercero y cuarto año, a quienes se les pidió que evaluaran el grado de veracidad y la pertinencia y claridad del vocabulario de los mismos. 
A partir de las modificaciones señaladas, se realizó un focus group con un grupo de expertos (oficiales instructores), quienes valoraron la calidad, veracidad y vocabulario técnico. Asimismo, se les solicitó que colocaran la mejor respuesta para cada situación relatada.

A partir de estas dos revisiones se construyó el instrumento piloto, conformado por 35 escenarios. El mismo fue contestado por 150 cadetes.

Posteriormente se procedió a realizar la categorización de las respuestas, las cuales fueron evaluadas por tres jueces en forma independiente, considerándose la fiabilidad de las puntuaciones interjueces (los valores se reseñan en el apartado de resultados).

Por último se eliminaron aquellos escenarios que eran de baja calidad, ya sea por haber obtenido una alta frecuencia de respuestas similares (falta de discriminación) o de baja calidad por parte de los 150 subtenientes. Finalmente el instrumento quedó conformado por 32 escenarios.

Esta última versión fue evaluada mediante un focus group de expertos (oficiales instructores), para ello se realizó una modificación en su formato (CTLM-E).

\section{Resultados}

A partir de las entrevistas, se detectaron 10 dimensiones o áreas de conocimiento. A continuación se reseñan fragmentos de las entrevistas vinculados a la dimensión: "Manejo de los errores propios frente al grupo". 
Inteligencia práctica: un instrumento para su evaluación

“... un oficial que habia cometido un error con un cadete de tercer año, le había gritado, lo había humillado. Y después resulta que por ahi estaba haciendo mal las cosas porque estaba enfermo. Y después se presenta y le dice me siento mal, estoy enfermo. Y el jefe le dijo me equivoqué. Y el cadete a pesar de su dolor, siguió trabajando.” “...yo comparto la idea de que un buen lider es aquel que comete un error y sabe reconocerlo delante de sus hombres".

“...estábamos en el terreno, estaba a cargo. El jefe nos delegaba muchas cosas para hacer y nos dividia mucho el trabajo. O sea que cada jefe de compañia tomaba sus decisiones y actuaba por su cuenta... Recibimos una serie de órdenes para tres dias seguidos, lo que ibamos a hacer hoy, lo que íbamos a hacer mañana, pasado. Cuando recibimos las órdenes esas, yo estaba muy cansado después de hacer todas las operaciones esas y empecé a copiar las ordenes desordenadas. Copiaba las órdenes que eran para dentro de dos dias para hoy entonces levanto a toda mi compañía, me fijo en el cronograma y les tocaba sesión de tiro nocturno que era en el polígono tres. El polígono tres estaba en otro lado, pero no lejos. Entonces me quedaba la duda porque no concordaban las cosas, y no sé si... si por miedo a preguntarle al jefe de la compañía porque él ya me lo había dado por no quedar como tonto, directamente voy hago levantar a mi compañia, hago armar el polígono en otro lado, sin consultar la orden que en realidad yo la había entendido mal. Los cadetes estaban cansados, venían de marchar, habian armado las carpas, estuvo lloviendo toda la noche. La cuestión es que hice correr a mi compañia cinco veces, asentaban las carpas y otra vez se tenían que correr. El jefe de la compañia me llama la atención lejos de la compañía y me dice yo le di claritas las órdenes, por que no me volvió a preguntar. Entonces fui, reuni a mi compañia, hablé con mis cadetes y reconoci mi error. Les dije cadetes vamos a tener que volver a movernos porque respondí 
mal a una orden que me dio el superior. Y al reconocer el error, a pesar del cansancio, todos continuaron trabajando hasta terminar".

La mayor parte de las dimensiones detectadas son coincidentes con el estudio realizado por Sternberg para el ejército norteamericano (Sternberg et al., 2000). Las diferencias se pueden apreciar en el Cuadro 2.

\section{Cuadro 2}

Áreas de conocimiento tácito según países

\begin{tabular}{|c|c|c|}
\hline $\begin{array}{l}\text { Tipo de } \\
\text { conocimiento } \\
\text { tácito }\end{array}$ & Muestra argentina & Muestra norteamericana \\
\hline Intrapersonal & $\begin{array}{l}\text { Manejo de los errores propios } \\
\text { frente al grupo. }\end{array}$ & Manejo del sí mismo. \\
\hline Intrapersonal & Selección de prioridades. & \\
\hline $\begin{array}{l}\text { Interpersonal: } \\
\text { Influenciar a otros }\end{array}$ & $\begin{array}{l}\text { Manejo de la información. } \\
\text { Combatir el rumor. }\end{array}$ & $\begin{array}{l}\text { Comunicación. Efectividad } \\
\text { con los soldados. }\end{array}$ \\
\hline $\begin{array}{l}\text { Interpersonal: } \\
\text { Influenciar a otros }\end{array}$ & $\begin{array}{l}\text { Mantener y elevar la moral } \\
\text { del grupo. }\end{array}$ & Motivar a los subordinados. \\
\hline Interpersonal: & Elección de subalternos para & Dirigir y supervisar. \\
\hline Influenciar a otros & armar grupos. & Armar grupos. \\
\hline $\begin{array}{l}\text { Interpersonal: } \\
\text { Influenciar a otros }\end{array}$ & Influenciar al jefe. & Influenciar al jefe. \\
\hline $\begin{array}{l}\text { Interpersonal: } \\
\text { Influenciar a otros }\end{array}$ & & $\begin{array}{l}\text { Uso del liderazgo para } \\
\text { resolver problemas. }\end{array}$ \\
\hline Interpersonal: & Establecer credibilidad para & Establecer credibilidad. \\
\hline Cooperar con otros & poder liderar. & Cooperar con otros. \\
\hline Organizacional & & $\begin{array}{l}\text { Comunicación: obtenerla } \\
\text { de los subordinados. }\end{array}$ \\
\hline Organizacional & $\begin{array}{l}\text { Manejo de errores de los } \\
\text { subordinados. }\end{array}$ & $\begin{array}{l}\text { Manejo de errores de los } \\
\text { subordinados. }\end{array}$ \\
\hline Organizacional & $\begin{array}{l}\text { Manejo de personas que no } \\
\text { responden bien. }\end{array}$ & $\begin{array}{l}\text { Trato con personas que no } \\
\text { responden bien. }\end{array}$ \\
\hline Organizacional & Manejo de sanciones. & $\begin{array}{l}\text { Manejo de los cambios } \\
\text { organizacionales. }\end{array}$ \\
\hline
\end{tabular}


Tal como fue reseñado, el instrumento piloto quedó conformado por 35 escenarios. A continuación se presenta un breve fragmento del instrumento a modo de ejemplo:

Situación 1: Transcurren los días previos a la licencia por Semana Santa. Como ocurre todos los años, durante la semana previa comienzan a circular rumores de que el franco estará supeditado al rendimiento de los cadetes. Los cadetes de 1er. año están ante su primer franco largo desde su incorporación. Ud. está a cargo de un pelotón de 1er. año y necesita cumplir con una tarea que le encomendaron relacionada con el desempeño de su pelotón, los integrantes del mismo están muy cansados y han llegado a esta instancia con pocas fuerzas para llevar a cabo la tarea con éxito. Las versiones son cada vez más fuertes y los cadetes se empiezan a desmotivar. ¿Cómo procede usted ante esta situación?

Situación 2: Está de maniobras. Debe elegir un subalterno para que esté a cargo de una patrulla durante la marcha. En su grupo tiene un cadete que está entre los primeros del orden de mérito, ha mostrado ser un cadete extremadamente capaz, pero Ud. no ha podido establecer un buen vínculo con él. Tiene otro cadete con rendimiento medio, pero destacado en su capacidad de mando y con el cual se ha establecido una buena relación. ¿Qué cadete elige para dirigir al subgrupo? ¿Por qué?

Situación 3: Su superior le ha encomendado una tarea que le insumirá todo el mes. Deberá arreglar la sala histórica, pintando, poniendo en condiciones los cuadros y consiguiendo los cuadros faltantes. Tiene los materiales necesarios. Sabe que si trabaja en forma constante podrá cumplir el objetivo. Pero también sabe que no puede bajar su rendimiento académico ni militar. ¿Cómo se organizará para cumplir esta tarea? 
El instrumento piloto fue contestado por 150 subtenientes. Posteriormente se procedió a realizar la categorización de las respuestas. A partir de las respuestas dadas por los expertos se diseñaron tres categorías: respuesta exitosa y completa $=2$ puntos, respuesta incompleta $=1$ punto, respuesta incorrecta $=0$ punto. Estas respuestas debían coincidir con las respuestas criterio obtenidas en el focus group de expertos (oficiales instructores).

Las mismas fueron trabajadas mediante el sistema de jueces. Tres jueces psicólogos que conocían la teoría de Sternberg realizaron una categorización a ciegas y posteriormente se analizó la confiabilidad interjueces. Ésta fue calculada mediante tres índices diferentes: 1) Confiabilidad entre los calificadores: de .83 a .97 (Sampieri et al., 1998); 2) Índice Kappa: .50 a .81 (Cohen, 1988), y 3 ) Coeficiente de Kendall de .63 a .92. En aquellos escenarios en los que no existió acuerdo se procedió a consensuar la mejor respuesta y se analizaron las discrepancias entre los jueces.

A continuación se expone un escenario con dos ejemplos de respuesta: una buena (a) y otra de baja calidad (b).

Situación 4: Está de maniobras. Debe elegir un subalterno para que esté a cargo de una patrulla durante la marcha. En su grupo tiene un cadete que está entre los primeros del orden de mérito, ha mostrado ser un cadete extremadamente capaz, pero Ud. no ha podido establecer un buen vinculo con él. Tiene otro cadete con rendimiento medio, pero destacado en su capacidad de mando y con el cual se ha establecido una buena relación. ¿Qué cadete elige para dirigir al subgrupo? ¿Por qué?

a) Elijo al cadete de rendimiento medio, al más idóneo y confiable. Lo hago porque tiene una buena capacidad de mando, porque es capaz de controlar al grupo, porque entenderá mi intención y sus órdenes serán mejor impartidas. 
b) Elijo al más capaz, si es el más capaz (uno de los primeros en orden de mérito) no debe haber inconvenientes. No deben influir los problemas de vínculo.

Posteriormente se realizó una versión para expertos (CTMLE), para ello se modificó el formato, se realizó una versión más fácil de evaluar (tanto para los expertos como para el equipo de investigación). Se redactó el escenario condensando el material en condiciones antecedentes, la situación dilemática y consecuencias (las respuestas de mejor performance). Para ello se utilizaron las preposiciones "Si ..." (para las antecedentes), "Y..." (para describir la situación) y "Entonces..." (para las respuesta- consecuencia).

Se solicitó a 10 oficiales instructores que los contestaran. A continuación se presenta un breve fragmento de la versión para oficiales:

Situación 1: Si Ud. está a cargo de un pelotón y comienzan a circular fuertes rumores respecto de la posibilidad de franco $\mathbf{Y}$ este franco está supeditado al rendimiento de los cadetes $\mathbf{Y}$ los cadetes están muy cansados para llevar adelante la tarea con éxito y están cada vez mas desmotivados, Entonces Ud. bloquea el rumor ya que no hay información oficial sobre el franco $\mathbf{Y}$ trata de motivar a los cadetes para la realización de las tareas mediante diversos métodos.

\begin{tabular}{|l|l|}
\hline $\begin{array}{l}\text { 1. El consejo de la situación me parece } \\
\text { muy bueno para la enseñanza del mando. }\end{array}$ & $\begin{array}{l}\text { Muy en Desacuerdo } 1-2-3-4-5 \\
\text { Muy de Acuerdo }\end{array}$ \\
\hline $\begin{array}{l}\text { 2. Lo relatado se enseña de forma explícita } \\
\text { en el CMN. }\end{array}$ & $\begin{array}{l}\text { Muy en Desacuerdo } 1-2-3-4-5 \\
\text { Muy de Acuerdo }\end{array}$ \\
\hline $\begin{array}{l}\text { 3. La solución del problema me parece } \\
\text { muy apropiada. }\end{array}$ & $\begin{array}{l}\text { Muy en Desacuerdo } 1-2-3-4-5 \\
\text { Muy de Acuerdo }\end{array}$ \\
\hline
\end{tabular}

Situación 2: Si Ud. está de maniobras y debe elegir un subalterno para que esté a cargo de una patrulla durante la marcha. Y 
en su grupo tiene un cadete que está entre los primeros del orden de mérito, pero Ud. no ha podido establecer un buen vínculo con él y hay otro cadete con rendimiento medio, pero destacado en su capacidad de mando y con el cual Ud. no tiene una buena relación. Entonces elige para dejar a cargo del grupo al cadete de rendimiento medio porque tiene una buena capacidad de mando $\mathbf{y}$ porque entenderá su intención y sus órdenes serán mejor impartidas.

\begin{tabular}{|l|l|}
\hline $\begin{array}{l}\text { 1. El consejo de la situación me parece } \\
\text { muy bueno para la enseñanza del mando. }\end{array}$ & $\begin{array}{l}\text { Muy en Desacuerdo } 1-2-3-4-5 \\
\text { Muy de Acuerdo }\end{array}$ \\
\hline $\begin{array}{l}\text { 2. Lo relatado se enseña de forma explícita } \\
\text { en el CMN. }\end{array}$ & $\begin{array}{l}\text { Muy en Desacuerdo } 1-2-3-4-5 \\
\text { Muy de Acuerdo }\end{array}$ \\
\hline $\begin{array}{l}\text { 3. La solución del problema me parece } \\
\text { muy apropiada. }\end{array}$ & $\begin{array}{l}\text { Muy en Desacuerdo } 1-2-3-4-5 \\
\text { Muy de Acuerdo }\end{array}$ \\
\hline
\end{tabular}

Situación 3: Si está en el Colegio Militar estudiando para rendir los exámenes y su oficial instructor le ha ordenado una tarea que le demandará la semana completa. Y la semana próxima serán todos los exámenes y en algunas materias en las cuales está muy atrasado sabe que no puede reprobar ninguna. Entonces habla con el oficial instructor y le plantea la situación y da prioridad a lo académico.

\begin{tabular}{|l|l|}
\hline $\begin{array}{l}\text { 1. El consejo de la situación me parece } \\
\text { muy bueno para la enseñanza del mando. }\end{array}$ & $\begin{array}{l}\text { Muy en Desacuerdo } 1-2-3-4-5 \\
\text { Muy de Acuerdo }\end{array}$ \\
\hline $\begin{array}{l}\text { 2. Lo relatado se enseña de forma explícita } \\
\text { en el CMN. }\end{array}$ & $\begin{array}{l}\text { Muy en Desacuerdo } 1-2-3-4-5 \\
\text { Muy de Acuerdo }\end{array}$ \\
\hline $\begin{array}{l}\text { 3. La solución del problema me parece } \\
\text { muy apropiada. }\end{array}$ & $\begin{array}{l}\text { Muy en Desacuerdo } 1-2-3-4-5 \\
\text { Muy de Acuerdo }\end{array}$ \\
\hline
\end{tabular}

Esta fase permitió evaluar la validez de contenido, la misma implica que el contenido del instrumento sea relevante y representativo para el constructo que se está evaluando. Para ello se formularon las preguntas a los expertos: 
- La calidad de ítem: cuán bueno es, qué grado de relevancia tiene para el conocimiento tácito, también se consideró que la respuesta de los cadetes presente cierta varianza (El consejo de la situación me parece muy bueno para la enseñanza del mando).

- Alta frecuencia de aparición de la situación y baja frecuencia de la respuesta óptima: cuando la situación no pudo generalizarse a los diferentes grupos (armas), cuando la respuesta reseñada no fue considerada buena o fue siempre la misma, se eliminó el ítem por bajo nivel de generalización, baja calidad y/o dificultad para discriminar (La solución del problema me parece muy apropiada).

- Nivel de representatividad del constructo: si eliminaron aquellos ítems que resultaron ser demasiado obvios, explícitos, limitados o técnicos (Lo relatado se enseña de forma explícita en el $C M N)$.

A partir de las respuestas obtenidas, se eliminaron aquellos escenarios en los que no había acuerdo o fueron puntuados como enseñanza de baja calidad para la formación del mando, escenario y respuesta poco implícita o respuesta de baja calidad.

\section{Discusión}

El presente trabajo intentó reseñar los pasos para la creación de un instrumento dirigido a evaluar la inteligencia práctica de cadetes militares utilizando un formato de lápiz y papel. Se encontraron similitudes en las dimensiones de conocimiento tácito para ámbitos militares detectadas por Sternberg para el ejército norteamericano y los datos presentados (Sternberg et al., 2000). Asimismo arribamos a una clara evidencia de la validez de contenido del instrumento diseñado. 
La construcción de instrumentos locales permite la adaptación a las particularidades y características culturales de cada medio. Entre las adaptaciones necesarias identificamos las referidas al contenido específico y las relativas al formato de respuesta abierto. En cuanto a la primera resultó necesario contar con personal experto que pudiera informar y adecuar los escenarios a un lenguaje militar de modo que fuera comprensible y que la prueba tuviera al mismo tiempo validez aparente. En cuanto a lo segundo, si bien Sternberg propone una versión final del instrumento para ámbitos militares- con una modalidad de respuesta basada en 10 opciones de respuesta, se decidió, en virtud de las características diferenciales de la muestra argentina, no utilizar formato forzado con tantos distractores. En función de ello se propuso un formato de respuesta abierta para categorizar las respuestas. Como inconveniente esta modalidad presenta el dificultoso procedimiento para la categorización de las respuestas provistas por los sujetos.

La evaluación del conocimiento tácito tiene la desventaja de ser poco generalizable y de requerir instrumentos específicos para cada población particular, dado que posee un gran componente técnico que es generalmente aprendido a partir de procesos de aprendizaje implícito. Como ventaja cabe citar la alta validez ecológica y el mejor poder de predicción del dominio específico del que se trate.

El instrumento que se presenta se encuentra en pleno proceso de construcción, aún restan evaluar sus características psicométricas específicas. Futuras investigaciones deberán examinar como medida de fiabilidad el grado de acuerdo entre jueces externos (expertos en el dominio) para proceder a la puntuación precisa de los escenarios y la validez de criterio del instrumento en su conjunto. El procedimiento de validación externa resultará de fundamental importancia para asegurar que el conocimiento tá- 
cito obtenido a través de la experiencia sea un buen predictor de liderazgo militar de los cadetes.

\section{Referencias}

Anastasi, A. (1998). Tests psicológicos (7a. ed.). México, D. F.: Prentice Hall.

Andrés Pueyo, A. (1997). Manual de psicología diferencial. Madrid: McGraw-Hill.

Berg, C. \& Calderone, K. (1994). The role of problem interpretation in understanding the development of everyday problem solving. En R. Sternberg \& W. Wagner (Eds.), Mind in context. Nueva York: Cambridge University Press.

Berry, J. W. \& Bennett, J. A. (1992). The conceptions of cognitive competence. International Journal of Psychology, 27, 73-88.

Boyatzis, R. E. (1982). The competent manager. Nueva York: John Wiley \& Sons.

Brody, N. (2000). History of theories and measurement of intelligence. En R. Sternberg (Ed.), Handbook of intelligence. Cambridge, Reino Unido: Cambridge University Press.

Carroll, J. B. (1993). Human cognitive abilities: A survey of factor-analytic studies. Cambridge, Reino Unido: Cambridge University Press.

Carroll, J. B. (1996). A three-stratum theory of intelligence: Spearman's contributions. En I. Dennis \& P. Tapsfield (Eds.), Human abilities: Their nature and measurement. Mahwah, NJ: Lawrence Erlbaum.

Cattell, R. B. (1943). The measurement of adult intelligence testing. Psychological Bulletin, 40, 153-193.

Cattell, R. B. (1963). Theory of fluid and crystallized intelligence: A critical experiment. Journal of Educational Psychology, 54, 1-22.

Ceci, S. J. (1996). On intelligence: A bioecological treatise on intellectual development. Cambridge, MA: Harvard University Press. 
Das, J. P. (1994). Eastern views of intelligence. En R. Sternberg (Ed.), Encyclopedia of human intelligence. Nueva York: Macmillan.

Davidson, J. E. \& Downing, C. L. (2000). Contemporary models of intelligence. En R. Sternberg (Ed.), Handbook of intelligence. Cambridge, Reino Unido: Cambridge University Press.

Flanagan, J. (1954). The critical incident technique. Psychological Bulletin, 51, 327-358.

Forsythe, G., Hedlund, J., Snook, S., Horvath, J., Williams, W., Bullis, R. et al. (1998, abril). Construct validation of tacit knowledge for military leadership. Presentado en el Encuentro Anual de la Asociación Americana de Investigadores de la Educación, San Diego, CA, EE. UU.

Gardner, H. (1983). Frames of mind: The theory of multiple intelligences. Nueva York: Basic Books.

Gardner, H. (1998). Inteligencias múltiples. La teoría en la práctica. Barcelona: Paidós.

Gottfredson, L. (1997). Why g matters: The complexity of everyday life. Intelligence, 24, 1, 79-132.

Gross, R. (1998). Psicología: la ciencia de la mente y la conducta. México: Manual Moderno.

Herrnstein, R. J. \& Murria, C. (1996). The Bell Curve. Nueva York: Free Press Paperback.

Irvine, S. H. \& Berry, J. W. (1986). The abilities of mankind: A reevaluation. En S. H. Irvine \& J. W. Berry (Eds.), Human abilities in cultural context. Nueva York: Cambridge University Press.

Ittenbach, R., Esters, I. \& Wainer, H. (1997). The history of test development. En D. Flanagan, J. Genshaft \& P. Harrison (Eds.), Contemporary intellectual assessment: Theories, tests, and issues. Nueva York: Guilford Press.

Mayer, J., Salovey, P. \& Caruso, D. (2000). Emotional intelligence. En R. Sternberg (Ed.), Handbook of intelligence. Cambridge, Reino Unido: Cambridge University Press. 
Myers, D. (1997). Psicología. Madrid: Panamericana.

Sampieri Hernández, R., Collado Fernández, C. \& Lucio Baptista, P. (1998). Metodología de la investigación. México: McGraw-Hill.

Sternberg, R. J. (1985). Beyond IQ: A triarchic theory of human intelligence. Nueva York: Cambridge University Press.

Sternberg, R. J. (1988). The triarchic mind: A new theory of human intelligence. Nueva York: Cambridge University Press.

Sternberg, R. J. (1997). Inteligencia exitosa. Barcelona: Piadós.

Sternberg, R. J. (1997a). The triarchic theory of intelligence. En

D. Flanagan, J. Genshaft \& P. Harrison (Eds.), Contemporary intellectual assessment: Theories, tests, and issues. Nueva York: Guilford Press.

Sternberg, R. J. (1998). Abilities as forms of developing expertise. Educational Researcher, 27, 11-20.

Sternberg, R. J. (2000). The concept of intelligence. En R. Sternberg (Ed.), Handbook of intelligence. Cambridge, Reino Unido: Cambridge University Press.

Sternberg, R. J. \& Detterman, D. (1992). ¿Qué es la inteligencia? Enfoque actual de su naturaleza y definición. Madrid: Pirámide.

Sternberg, R. J., Forsythe G. B., Hedlund, J., Horvath, J., Snook, S., Williams W. M. et al. (2000). Practical intelligence in everyday life. Nueva York: Cambridge University Press.

Sternberg, R. J., Lautrey, J. \& Lubart, T. (2003). Models of intelligence: International Perspectives. Washington DC: American Psychological Association.

Sternberg, R. J., Wagner, R. \& Okagaki, L. (1993). Practical intelligence: The nature and role of tacit knowledge in work and school. En H. Reese \& J. Puckett (Eds.), Advances in lifespan development. Hillsdale, NJ: Erlbaum.

Sternberg, R. J., Wagner, R. K., Williams, W. M. \& Horvath, J. A. (1995). Testing common sense. American Psychologist, 50, $11,912-927$. 
Thorndike, R. M. (1997). The early history of intelligence testing. En D. Flanagan, J. Genshaft \& P. Harrison (Eds.), Contemporary intellectual assessment: Theories, tests, and issues. Nueva York: Guilford Press.

Wagner, R. K. (1987). Tacit knowledge in everyday intelligent behavior. Journal of Personality and Social Psychology, 52, 6, 1236-1247.

Wagner, R. K. (1994). Practical intelligence. European Journal of Psychological Assessment, 10, 2, 162-169.

Wagner, R. K. (2000). Practical intelligence. En R. Sternberg (Ed.), Handbook of intelligence. Cambridge, Reino Unido: Cambridge University Press.

Wagner, R. K. \& Sternberg, R. J. (1985). Practical intelligence in real-world pursuits: The role of tacit knowledge. Journal of Personality and Social Psychology, 48, 436-548.

Wolf, T. H. (1973). Alfred Binet. Chicago: University of Chicago Press. 